\title{
Myelodysplastic Syndrome and Sweet's Syndrome Are Associated with a Mutation in Isocitrate Dehydrogenase 1
}

\author{
ROSE SNYDER ${ }^{1}$, TIFFANY LIBBY ${ }^{2}$, PATRICIA RACITI ${ }^{2}$, BIJAL AMIN ${ }^{2}$, \\ MARK JACOBSON ${ }^{2}$, DINESH RAKHEJA ${ }^{3}$, KIRSTEN FLEMING ${ }^{2}$, MATTHIAS BARTENSTEIN ${ }^{4}$, \\ CHANGCHENG ZHU ${ }^{5}$, SWATI GOEL ${ }^{4}$, AMIT K. VERMA ${ }^{4}$ and ADITI SHASTRI ${ }^{4}$ \\ Departments of ${ }^{1}$ Medicine, ${ }^{2}$ Dermatology, ${ }^{4}$ Hemato-Oncology, and ${ }^{5}$ Pathology, \\ Albert Einstein College of Medicine, Bronx, NY, U.S.A.; \\ ${ }^{3}$ Department of Pathology, UT Southwestern Medical Center, Dallas, TX, U.S.A.
}

\begin{abstract}
Background: Sweet's syndrome (SS) is a febrile neutrophilic dermatosis that has been clinically linked to hematological malignancies, particularly myelodysplastic syndrome (MDS), in a number of case series. Many epigenetic changes underlying MDS have been identified, such as a mutation in the isocitrate dehydrogenase 1 (IDHI) gene, which causes DNA hypermethylation and alteration of a number of genes that lead to leukemogenesis. However, the pathogenesis of malignancy-associated SS is unknown. Case Report: We present two patients who were diagnosed with SS and concomitant IDHI-mutated MDS. Immunohistochemical staining of their skin lesions showed neutrophils diffusely positive for the IDHI mutation. Conclusion: These cases demonstrate that IDHI mutation may be implicated in the pathogenesis of malignancy-associated SS. Future investigation to elucidate this pathway is warranted. Establishing this molecular link can provide an earlier identification of patients with SS who are also at increased risk for developing MDS.
\end{abstract}

Sweet's syndrome (SS) was first described in 1964 as an acute febrile neutrophilic dermatosis. It presents clinically with tender nodules that consist histopathologically of a heavy neutrophilic infiltrate in the subcutis as well as dermis (1). A variant of this condition called histiocytoid SS has a similar clinical presentation and includes infiltration of immature myeloid cells that morphologically resemble histiocytes and stain positively for myeloperoxidase (2). SS has been

This article is freely accessible online.

Correspondence to: Aditi Shastri, MD, Albert Einstein College of Medicine, 1300 Morris Park Avenue, Chanin Building, Room 302B, Bronx, NY 10461, U.S.A. E-mail: ashastri@montefiore.org

Key Words: Sweet's syndrome, myelodysplastic syndrome, IDHI mutation. clinically associated with myeloid malignancies including acute myeloid leukemia (AML) and myelodysplastic syndromes (MDS) (3-8). The pathophysiology of malignancyassociated SS is largely unknown and there are no established guidelines regarding the treatment of this condition (9). Typically, SS responds to treatment with corticosteroids and treatment of the underlying myeloid malignancy.

MDS is a heterogeneous group of hematological disorders that are characterized by cytopenia due to ineffective hematopoiesis and confer an increased risk of development of AML. Isocitrate dehydrogenase (IDH) proteins are homodimeric enzymes that are involved in important cellular processes of histone and DNA modification as well as the cellular response to hypoxia (10). Recurring $I D H I$ gene mutations have been detected in $20 \%$ of adults with AML and $5 \%$ of adults with MDS $(11,12)$.The IDH1 and IDH2 enzymes, under normal circumstances, catalyze the oxidative decarboxylation of isocitrate to $\alpha$-ketoglutarate $(\alpha-K G)$. Mutant IDH enzymes however, catalyze the reduction of $\alpha-K G$ to the onco-metabolite D-2-hydroxy-glutarate (D$2 \mathrm{HG}$ ), resulting in numerous epigenetic aberrations that include DNA hypermethylation and altered expression of many important genes, leading to leukemogenesis $(11,13)$.

A clinical association between SS and MDS has been described in a number of reports. However, the molecular pathogenesis underlying this presentation has not yet been elucidated $(7,14)$. Here we present two patients with SS and underlying IDHI-mutated MDS whose skin biopsies stained positively for $I D H I$-mutant neutrophils. We discuss IDHI mutation as a possible molecular link between these two syndromes that can be treated by targeted therapy such as the IDH inhibitors.

\section{Case Reports}

Case 1: A 50-year-old man presented with multiple subcutaneous nodules on his chest, upper and lower extremities 

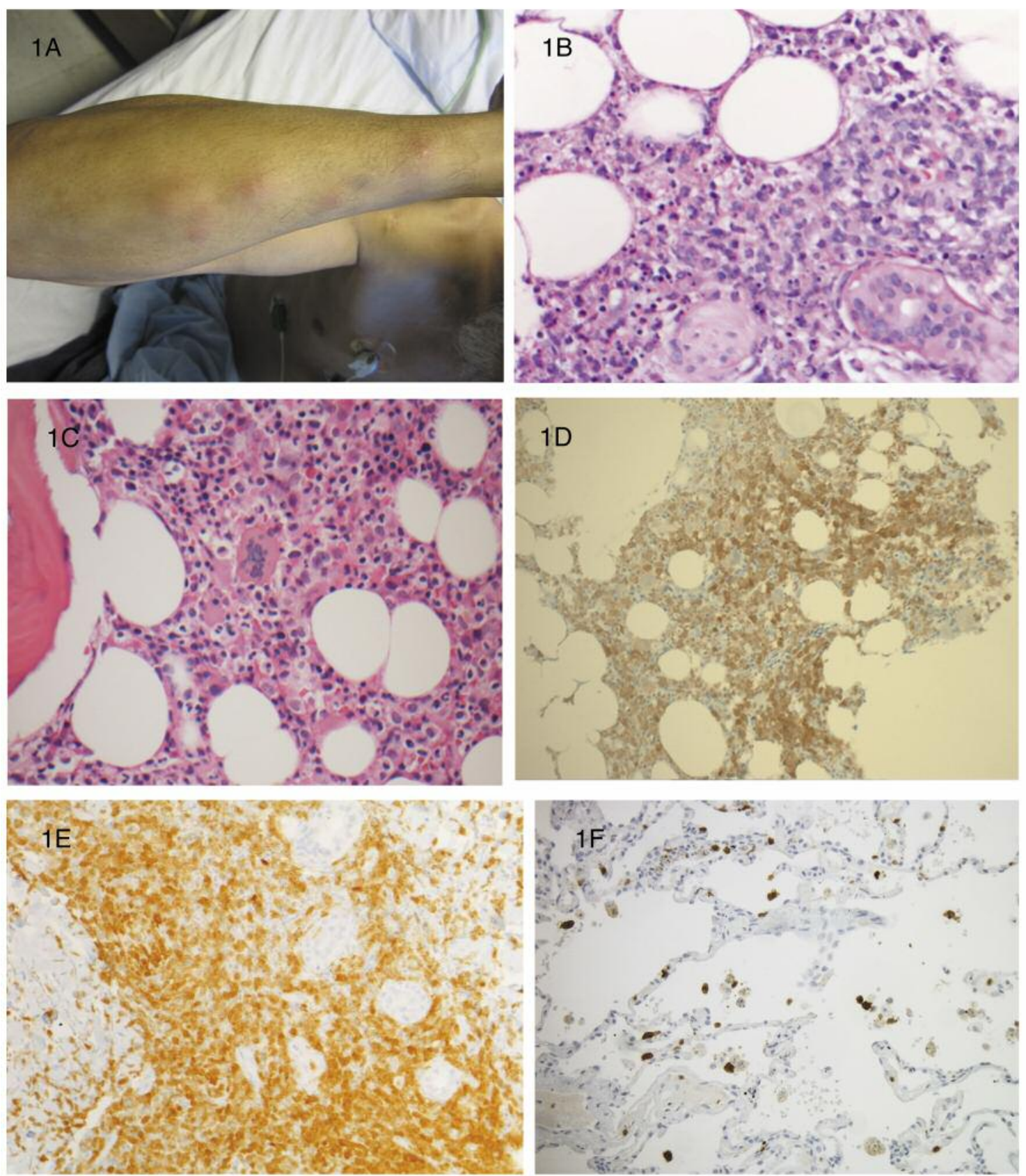

Figure 1. Biopsy findings in case 1. A: Tender, erythematous and edematous subcutaneous nodules on the dorsum of patient 1 arm. These nodules were present on his chest, upper and lower extremities. B: Skin nodule biopsy showing an infiltrate composed of many neutrophils, including immature neutrophils resembling histiocytes, which was confirmed by myeloperoxidase and CD68 stains (not shown). Hematoxylin and Eosin, magnification 40x. C: Bone marrow biopsy showing slightly hypercellular marrow and with a dysplastic megakaryocyte. D: Immunohistochemical (IHC) staining for the isocitrate dehydrogenase 1 (IDH1) mutation of the bone marrow showing positive staining in myelomonocytic cells and megakaryocytes. Magnification 20x. E: IHC staining for the IDH1 mutation on the skin biopsy showing neutrophilic infiltrate diffusely positive for the IDH1 mutation. F: IHC staining for the IDH1 mutation of the lung parenchyma showing positive staining in macrophages.

that were erythematous, edematous and mildly tender to palpation (Figure 1A). His medical record showed a history of development of unexplained febrile neutropenia and similar skin findings during the year prior to this admission. Punch biopsy of a skin lesion on the right arm was performed and showed subcutaneous histiocytoid SS (Figure 1B). Within weeks of this presentation, he was found to have neutropenia and macrocytic anemia on routine blood tests. His complete blood count 

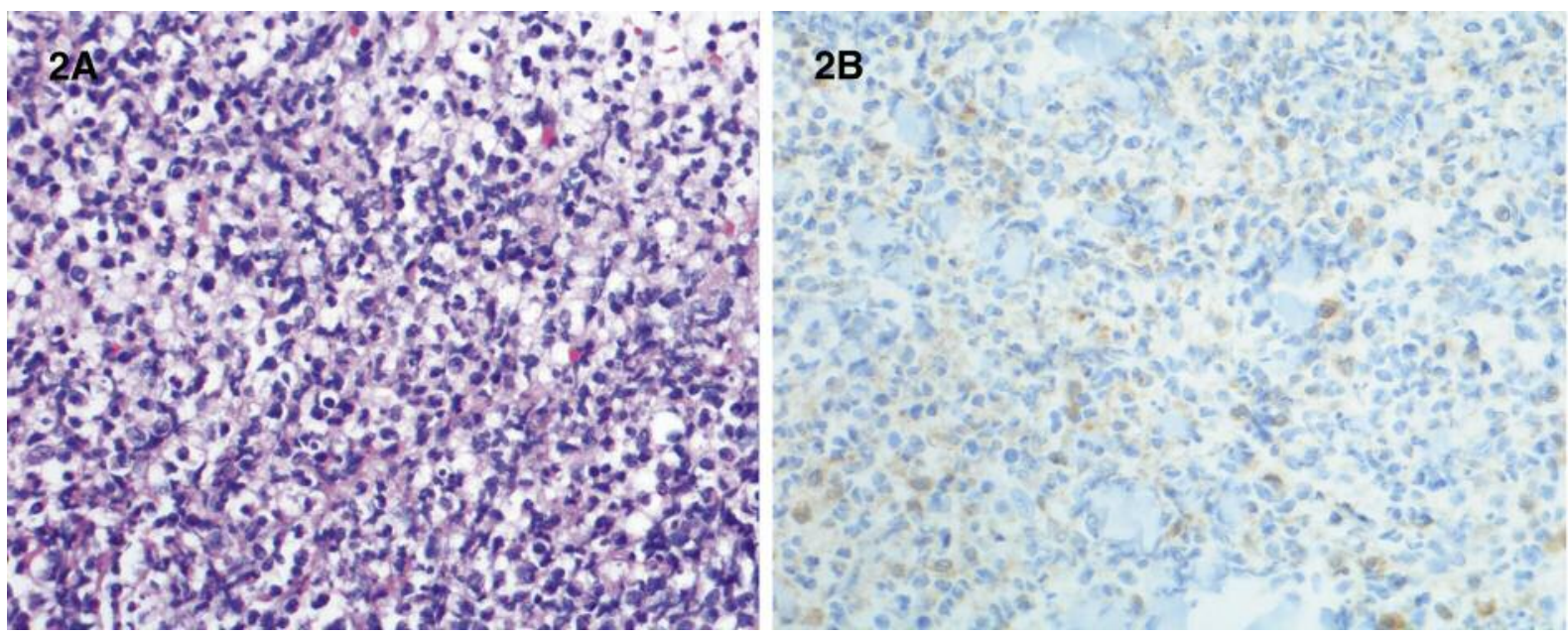

Figure 2. Biopsy findings in case 2. A: Skin nodule biopsy showing a dense neutrophilic infiltrate. Hematoxylin and Eosin, 40x. B: Immunohistochemical staining for the isocitrate dehydrogenase 1 (IDH1) mutation on the skin biopsy showing neutrophilic infiltrate positive for the IDH1 mutation. Magnification 40x.

demonstrated a hemoglobin of $9.2 \mathrm{~g} / \mathrm{dl}$, hematocrit of 31.3 , white blood cell count of $2.5 \times 10^{9} / 1$, platelets of $252 \times 10^{9} / 1$, and a mean corpuscular volume (MCV) of $100 \mathrm{fl}$. Subsequently, the patient underwent a bone marrow biopsy for pancytopenia, which revealed a diagnosis of intermediate-risk MDS by the International Prognostic Scoring System criteria (15) (Figure 1C). Molecular profiling of the bone marrow aspirate with a 44myeloid gene mutational panel showed somatic mutations in the IDHI gene (c.395G >A; p.R132H) with an allelic frequency of $35 \%$. It also showed a somatic mutation in the splicing factor Zinc finger $\mathrm{CCCH}$-type, RNA binding motif and serine/arginine rich 2 (ZRSR2) gene (c.263_266delTAAA; frameshift). Immunohistochemical staining (IHC) of the bone marrow biopsy was positive for the IDHI mutation in myelomonocytic cells and megakaryocytes (Figure 1D). IHC of a lesion of the right upper extremity demonstrated the majority of infiltrating neutrophilic cells also to be positive for the IDHI (R132H) mutation (Figure 1E). A serum sample from the patient was analyzed for D-2HG level, which was found to be significantly elevated at $1438 \mathrm{ng} / \mathrm{ml}$ (reference range: $18-263 \mathrm{ng} / \mathrm{ml}$ ).

The patient was treated with $40 \mathrm{mg}$ of oral prednisone for SS. He experienced rapid improvement of his skin lesions and resolution of fever. The skin lesion recurred and the patient underwent seven cycles of treatment with a hypomethylating agent, azacitidine, which is the first-line therapy for higher risk MDS. Treatment with azacitidine resulted in improvement of anemia. The skin lesions only partially improved and the patient required numerous cycles of prednisone, with temporary resolution. The patient subsequently developed IDHI-mutated neutrophilic infiltration in the lungs (Figure 1F), and after multiple prolonged hospitalizations was transferred to hospice care with multi-organ failure.
Case 2: A 73-year-old male was referred to the hematology clinic for persistent leukopenia and anemia. He had a history of SS diagnosed 4 years prior based on skin biopsy (Figure 2A). He experienced intermittent relapse of SS characterized by painful erythematous and edematous nodules on his hands, usually treated with prednisone. Recurrence was often associated with fever, leukocytosis, and myalgia. At the time of presentation to the hematology clinic, his complete blood count demonstrated: hemoglobin $10 \mathrm{~g} / \mathrm{dl}$, hematocrit 32.4, white blood cell count $3.4 \times 10^{9} / 1$, platelets $148 \times 10^{9} / 1$, with $\mathrm{MCV}$ of $95.3 \mathrm{fl}$.

The patient underwent a bone marrow biopsy, which showed a hypercellular marrow (50-60\%) with trilineal hematopoiesis, mild erythroid hyperplasia, mild dysplasia and no significant increase in blasts. Molecular profiling of the bone marrow aspirate showed somatic mutations in the $\mathrm{IDH} I$ gene (c.395G >A; p.R132H) with an allelic frequency of $45 \%$. It also showed a somatic mutation in the serine and arginine rich splicing factor 2 (SRSF2) gene (c.284C >A;p.P95H) with an allelic frequency of $46 \%$. A diagnosis of low-grade MDS was made. On immunohistochemical staining, the skin biopsy showed the neutrophilic infiltrate to be positive for the IDHI mutation (Figure 2B). The patient required close monitoring, but no acute intervention for MDS.

\section{Discussion}

Sweet's syndrome and the histiocytoid variant have been associated with myeloid malignancies. Some reports have estimated the incidence of SS and concomitant hematological malignancy to be as high as $18 \%(16,17)$. MDS, in particular, appears to have a unique association with SS, with the suggestion of a higher association of the histiocytoid variant 
rather than conventional SS with $\operatorname{MDS}(18,19)$. In one case series, $31.8 \%$ of patients with histiocytoid SS were diagnosed with MDS (19). A distinct chronic subset of SS has also been described that is often associated with fever and arthralgia. A number of reports have suggested that this atypical presentation of SS is closely associated with the later development of MDS (18, 20, 21). The time lag between development of skin findings and diagnosis of MDS in these cases was 0-7 years $(18,21)$. For both our patients, their skin manifestations and diagnosis of SS predated their diagnosis of MDS, and this fits the pattern as previously described in the literature. This presentation highlights the importance of working up a patient that presents with SS for an underlying hematologic malignancy. In cases where the skin findings are chronic and associated with constitutional symptoms, this may suggest a more specific association of SS and MDS.

Epigenetic mutations and genetic dysfunction are thought to play a significant role in the pathogenesis of MDS. Patients with mutant $I D H 1 / 2$ exhibit a global hypermethylation phenotype associated with significant suppression of gene expression compared to patients without IDH-mutated myeloid malignancies (10). The presence of $I D H 1 / 2$ mutation correlates well with elevated serum D-2HG in patients with AML (22). Mutant IDH1/2 proteins catalyze the reduction of $\alpha-K G$ to $D-2 H G$, which is structurally similar to $\alpha-K G$ and has been shown to competitively inhibit $\alpha$-KG-dependent enzymes. Important among these are the ten-eleventranslocation (TET) 2 enzyme and histone lysine demethylases. TET2 is thought to cause DNA demethylation by genome-wide, as well as locus-specific, hydroxymethylation. The histone demethylase enzymes are important for regulating the process of gene transcription by chromatin modifications (11). Compared to the significant amount of information available about mutant IDH1/2 in MDS, almost nothing is known about the pathobiology of malignancyassociated SS. Recently, a presentation of SS and concomitant MDS was reported in association with a heterozygous mutation in the $M E F V$ gene, associated with familial Mediterranean fever, with high fever and marked neutropenia (23). Another report noted an increase in interleukin-1 $\beta$ expression within the SS skin lesions (24). This leads us to speculate that alterations in immune signaling play a large role in the pathophysiology of SS. Future studies directed at the association of mutant IDH1 and immune signaling in SS will help forward our understanding of this association. The presence of an elevated level of D-2HG as well as multi-organ involvement with IDH1-mutated immune cells in our first patient strongly suggests that $I D H 1$ mutations may dysregulate a myriad of cellular pathways and functions beyond its known effects that are causative of hematological malignancies. In summary, we strongly feel that IDHI mutations may predispose to a global dysfunction that extends beyond its known effects in dysregulated hematopoiesis.

\section{Conclusion}

We presented two cases of $I D H 1$-mutated MDS and SS. The IDH1 mutation (R132H) was seen in virtually all of the infiltrating neutrophils in the skin lesions which preceded the development of cytopenia and MDS. Establishing a new molecular link such as the IDHI mutation between SS and MDS can provide an earlier identification of patients presenting with SS who are also at increased risk for developing myeloid malignancies, particularly MDS. It can also guide patients to molecularly targeted therapies such as IDH inhibitors that are specific to the biology of their disease (25-27).

\section{References}

1 Sweet RD: An acute febrile neutrophilic dermatosis. $\mathrm{Br} \mathrm{J}$ Dermatol 76: 349-356, 1964.

2 Requena L, Kutzner H, Palmedo G, Pascual M, FernandezHerrera J, Fraga J, Garcia-Diez A and Yus ES: Histiocytoid sweet syndrome: A dermal infiltration of immature neutrophilic granulocytes. Arch Dermatol 141(7): 834-842, 2005.

3 Spector JI, Zimbler H, Levine R, Ross JS, Valigorsky JM and Cole LM: Sweet's syndrome. Association with acute leukemia. JAMA 244(10): 1131-1132, 1980.

4 Goodfellow A and Calvert H: Sweet's syndrome and acute myeloid leukaemia. Lancet 2(8140): 478-479, 1979.

5 Klock JC and Oken RL: Febrile neutrophilic dermatosis in acute myelogenous leukemia. Cancer 37(2): 922-927, 1976.

6 Caughman W, Stern R and Haynes H: Neutrophilic dermatosis of myeloproliferative disorders. Atypical forms of pyoderma gangrenosum and Sweet's syndrome associated with myeloproliferative disorders. J Am Acad Dermatol 9(5): 751-758, 1983.

7 Magro CM, Momtahen S, Nguyen GH and Wang X: Histiocytoid Sweet's syndrome: A localized cutaneous proliferation of macrophages frequently associated with chronic myeloproliferative disease. Eur J Dermatol 25(4): 335-341, 2015.

8 Chan MP, Duncan LM and Nazarian RM: Subcutaneous Sweet syndrome in the setting of myeloid disorders: A case series and review of the literature. J Am Acad Dermatol 68(6): 1006-1015, 2013.

9 Raza S, Kirkland RS, Patel AA, Shortridge JR and Freter C: Insight into Sweet's syndrome and associated-malignancy: A review of the current literature. Int J Oncol 42(5): 1516-1522, 2013.

10 Figueroa ME, Abdel-Wahab O, Lu C, Ward PS, Patel J, Shih A, Li Y, Bhagwat N, Vasanthakumar A, Fernandez HF, Tallman MS, Sun Z, Wolniak K, Peeters JK, Liu W, Choe SE, Fantin VR, Paietta E, Lowenberg B, Licht JD, Godley LA, Delwel R, Valk PJ, Thompson CB, Levine RL and Melnick A: Leukemic IDHI and $I D H 2$ mutations result in a hypermethylation phenotype, disrupt TET2 function, and impair hematopoietic differentiation. Cancer Cell 18(6): 553-567, 2010.

11 Medeiros BC, Fathi AT, DiNardo CD, Pollyea DA, Chan SM and Swords R: Isocitrate dehydrogenase mutations in myeloid malignancies. Leukemia 31(2): 272-281, 2017.

12 Dang L, White DW, Gross S, Bennett BD, Bittinger MA, Driggers EM, Fantin VR, Jang HG, Jin S, Keenan MC, Marks 
KM, Prins RM, Ward PS, Yen KE, Liau LM, Rabinowitz JD, Cantley LC, Thompson CB, Vander Heiden MG and Su SM: Cancer-associated $I D H 1$ mutations produce 2-hydroxyglutarate. Nature 465(7300): 966, 2010.

13 Im AP, Sehgal AR, Carroll MP, Smith BD, Tefferi A, Johnson DE and Boyiadzis $\mathrm{M}:$ DNMT3A and $I D H$ mutations in acute myeloid leukemia and other myeloid malignancies: Associations with prognosis and potential treatment strategies. Leukemia 28(9): 1774-1783, 2014.

14 Bush JW and Wick MR: Cutaneous histiocytoid Sweet syndrome and its relationship to hematological diseases. J Cutan Pathol 43(4): 394-399, 2016.

15 Sanz GF, Sanz MA and Greenberg PL: Prognostic factors and scoring systems in myelodysplastic syndromes. Haematologica 83(4): 358-368, 1998.

16 Alegria-Landa V, Rodriguez-Pinilla SM, Santos-Briz A, Rodriguez-Peralto JL, Alegre V, Cerroni L, Kutzner H and Requena L: Clinicopathologic, immunohistochemical, and molecular features of histiocytoid Sweet syndrome. JAMA Dermatol 153(7): 651-659, 2017.

17 Villarreal-Villarreal CD, Ocampo-Candiani $\mathrm{J}$ and VillarrealMartinez A: Sweet syndrome: A review and update. Actas Dermosifiliogr 107(5): 369-378, 2016.

18 Vignon-Pennamen MD, Juillard C, Rybojad M, Wallach D, Daniel MT, Morel P, Verola O and Janin A: Chronic recurrent lymphocytic Sweet syndrome as a predictive marker of myelodysplasia: A report of 9 cases. Arch Dermatol 142(9): 1170-1176, 2006

19 Ghoufi L, Ortonne N, Ingen-Housz-Oro S, Barhoumi W, Begon E, Haioun C, Pautas C, Beckerich F, Robin C, Wolkenstein P, Cordonnier C, Chosidow O and Toma A: Histiocytoid Sweet syndrome is more frequently associated with myelodysplastic syndromes than the classical neutrophilic variant: A comparative series of 62 patients. Medicine 95(15): e3033, 2016.

20 Wang YN, Zhuang JL, Zhao WL, Fang K, Liu YH, Jin HZ and Li L: [clinical analysis of Sweet syndrome with myelodysplasia syndrome]. Zhonghua Yi Xue Za Zhi 96(22): 1755-1757, 2016.

21 Kulasekararaj AG, Kordasti S, Basu T, Salisbury JR, Mufti GJ and du Vivier AW: Chronic relapsing remitting Sweet syndrome - a harbinger of myelodysplastic syndrome. Br J Haematol 170(5): 649-656, 2015.
22 DiNardo CD, Propert KJ, Loren AW, Paietta E, Sun Z, Levine RL, Straley KS, Yen K, Patel JP, Agresta S, Abdel-Wahab O, Perl AE, Litzow MR, Rowe JM, Lazarus HM, Fernandez HF, Margolis DJ, Tallman MS, Luger SM and Carroll M: Serum 2hydroxyglutarate levels predict isocitrate dehydrogenase mutations and clinical outcome in acute myeloid leukemia. Blood 121(24): 4917-4924, 2013.

23 Jo T, Horio K and Migita K: Sweet's syndrome in patients with MDS and MEFV mutations. N Engl J Med 372(7): 686-688, 2015.

24 Navarini AA, Satoh TK and French LE: Neutrophilic dermatoses and autoinflammatory diseases with skin involvement--innate immune disorders. Semin Immunopathol 38(1): 45-56, 2016.

25 Yen K, Travins J, Wang F, David MD, Artin E, Straley K, Padyana A, Gross S, DeLaBarre B, Tobin E, Chen Y, Nagaraja R, Choe S, Jin L, Konteatis Z, Cianchetta G, Saunders JO, Salituro FG, Quivoron C, Opolon P, Bawa O, Saada V, Paci A, Broutin S, Bernard OA, de Botton S, Marteyn BS, Pilichowska M, Xu Y, Fang C, Jiang F, Wei W, Jin S, Silverman L, Liu W, Yang H, Dang L, Dorsch M, Penard-Lacronique V, Biller SA and Su SM: Ag-221, a first-in-class therapy targeting acute myeloid leukemia harboring oncogenic $I D H 2$ mutations. Cancer Discov 7(5): 478-493, 2017.

26 Birendra $\mathrm{KC}$ and DiNardo $\mathrm{CD}$ : Evidence for clinical differentiation and differentiation syndrome in patients with acute myeloid leukemia and idh1 mutations treated with the targeted mutant IDH1 inhibitor, ag-120. Clin Lymphoma Myeloma Leuk 16(8): 460-465, 2016.

27 Stein EM, DiNardo CD, Pollyea DA, Fathi AT, Roboz GJ, Altman JK, Stone RM, DeAngelo DJ, Levine RL, Flinn IW, Kantarjian HM, Collins R, Patel MR, Frankel AE, Stein A, Sekeres MA, Swords RT, Medeiros BC, Willekens C, Vyas P, Tosolini A, Xu Q, Knight RD, Yen KE, Agresta S, de Botton S and Tallman MS: Enasidenib in mutant IDH2 relapsed or refractory acute myeloid leukemia. Blood 130(6): 722-731, 2017.

Received January 31, 2018

Revised February 26, 2018

Accepted February 27, 2018 